\title{
COMPATIBILIDAD DE LAS RUTINAS DE PENSAMIENTO CON EL APRENDIZAJE INTEGRADO DE CONTENIDOS Y LENGUA EXTRANJERA (AICLE): UN ESTUDIO EXPLORATORIO ${ }^{1}$
}

\author{
EXPLORING THE ROLE OF THINKING \\ ROUTINES IN CLIL CLASSROOMS
}

\author{
Alfonso López Hernández ${ }^{2}$ \\ https://orcid.org/0000-0001-8174-595X
}

Recibido: abril 19, 2020 - Aceptado: mayo 7, 2020.

\section{RESUMEN}

El presente estudio, de carácter exploratorio, aborda las posibilidades de las llamadas rutinas de pensamiento, originadas en el Proyecto Cero de la Universidad de Harvard, en contextos de Aprendizaje Integrado de Contenidos y Lengua Extranjera (AICLE; CLIL, en inglés), con enfoque de enseñanza bilingüe cada vez más común, tanto en Europa como en Latinoamérica. Se analiza, en primer lugar, la fundamentación teórica del pensamiento visible -marco conceptual del que surgen las rutinas-con especial atención a los puntos en común relacionados con el papel que la cognición juega en el enfoque AICLE. La segunda parte del estudio expone los resultados de la aplicación sistemática de las siete rutinas básicas o fundamentales con estudiantes de formación de profesorado de un centro universitario de Madrid (España), por un lado, y en tres escuelas de educación primaria, por otro; siempre en ámbitos de aprendizaje de los contenidos curriculares en lengua inglesa. Estas experiencias apuntan al gran valor de las rutinas como estrategia metodológica en el aula AICLE, en especial por su capacidad para fomentar la participación de todo el alumnado -independientemente de su competencia lingüística-, sobre todo a través de la formulación de preguntas. Sin embargo, también se señalan algunas limitaciones de esta estrategia, tanto en relación con su riqueza cognitiva como en su capacidad para generar situaciones de verdadera comunicación en el aula. Por último, se proponen una serie de preguntas de investigación para investigaciones futuras.

Palabras clave: AICLE, CLIL, educación bilingüe, Proyecto Cero, rutinas de pensamiento, pensamiento visible.

1 El autor de este artículo está en deuda con los profesores Rodrigo Ruiz Aguarón y Mercedes Maldonado Morales, que dirigieron la mayoría de las rutinas de pensamiento con estudiantes de educación primaria. Asimismo, agradece la disponibilidad de los centros CEIP Aldebarán, Virgen de Mirasierra y Mater Immaculata para poder entrar en sus clases a observar o dirigir la realización de dichas rutinas.

2 Doctor en Filosofía, Universidad Complutense de Madrid, España. Profesor colaborador asistente a tiempo completo. Coordinador de los estudios de Lengua Inglesa y AICLE en Educación. Facultad de Ciencias Humanas y Sociales, Universidad Pontificia Comillas, España. Página web personal: alfonsolopezhernandez.com alhernandez@comillas.edu 


\section{ABSTRACT}

This paper presents provides an initial assessment of the benefits that can be obtained by applying thinking routines (Project Zero, Harvard University) in contexts of Content and Language Integrated Learning (CLIL), an approach to bilingual education that is now commonplace in Europe and that is gaining ground in other continents. The first part of the study provides a discussion of the theoretical compatibility of visible thinking -the larger conceptual framework which supports the routines- and CLIL, which has the student's cognitive engagement as one of its central methodological principles. In turn, the second part presents the result of a systematic application of the 7 introductory or core routines with preservice teacher trainees in Madrid (Spain) studying a university CLIL program, as well as some experiences of these routines with Elementary education students in three schools of the region. All these experiences attest to the significant benefits of using thinking routines in bilingual instruction, especially in what regards a greater student involvement than alternative practices, on the one hand, and the ability to provide extensive oral practice in question forming. However, a number of their limitations is also discussed, specifically in relation to their cognitive quality as well as their ability to generate situations of authentic communication in L2. Based on these preliminary findings, specific questions for future studies are proposed.

Keywords: CLIL, Bilingual education, Project Zero, Thinking routines, Visible Thinking

\section{INTRODUCCIÓN}

En un contexto educativo marcado por el auge de las llamadas metodologías activas -sobre todo en educación primaria, pero también en secundaria y etapas postobligatorias-, se han popularizado en muchos países las estrategias metodológicas relacionadas con el pensamiento visible. Dicho concepto, que proporciona un marco conceptual y estratégico orientado a integrar el desarrollo del pensamiento de los estudiantes con el aprendizaje de las diferentes áreas curriculares, se asocia con el Proyecto Cero de la Universidad de Harvard, así como con autores como David Perkins, Ron Ritchart, Shari Tishman o, fuera del Proyecto Cero, Robert Swartz. La popularización de dicho marco conceptual se traduce, en la práctica, en la creciente utilización por parte de profesores y métodos editoriales, de estrategias tales como rutinas y destrezas de pensamiento, organizadores gráficos o actividades de metacognición.

Es interesante señalar que, en el panorama educativo actual, es común que la aplicación de estrategias de pensamiento tenga lugar en contextos de enseñanza bilingüe, en una lengua -normalmente el inglés- que los alumnos pueden estar lejos de dominar. Ciertamente, son cada vez más los sistemas educativos en Europa y América Latina que potencian la enseñanza bilingüe en la escuela pública y sostenida con fondos públicos, para reforzar la enseñanza de la lengua extranjera. En Europa, dicha apuesta ha adquirido, mayoritariamente, la forma del Aprendizaje Integrado en Contenidos y Lengua Extranjera (AICLE), conocido más generalmente por su acrónimo inglés, CLIL. AICLE es un llamado «término paraguas» (Mehisto et al., 2018), es decir, recoge distintos modelos en donde el aprendizaje curricular se integra con el aprendizaje de la lengua extranjera de formas diversas, tanto en lo relativo a las metodologías de enseñanza como al peso de los contenidos impartidos en L2, en el currículo educativo. Sin embargo, en términos esenciales. se trata de un enfoque de naturaleza «dual» (Coyle et al., 2010, p.1), es decir, atiende tanto al aprendizaje del contenido curricular como a la mejora de la competencia en la lengua adicional (Graddol, 2006, p. 86). 
Debido a este carácter dual, el enfoque AICLE difiere radicalmente de los modelos de inmersión lingüística en los que, por lo general, la competencia lingüística del alumno en la lengua meta se da por sentada $\mathrm{o}$, al menos, no se refuerza explícitamente en las materias curriculares no lingüísticas. Los programas AICLE, por el contrario, parten de la premisa de que ni los alumnos ni -en la mayoría de los casos- los docentes, tienen plena competencia en la lengua meta, por lo que se asume que el andamiaje (scaffolding) del aprendizaje en L2 y uso de L2 deben tener lugar desde las propias materias del currículo (Dale y Tanner, 2018, pp. 4-5). Es seguramente debido a ese mayor realismo que, a pesar de ser un modelo que surge en un contexto europeo, ha sido exportado con éxito a sistemas educativos y colegios en Asia o América Latina (ver, por ejemplo, el caso de Colombia en [McDougald, 2015]).

Por consiguiente, el presente estudio aborda la compatibilidad del pensamiento visible con el AICLE y, en especial, el posible encaje de las rutinas de pensamiento en el aula bilingüe. Las rutinas de pensamiento han sido definidas como «una serie corta de preguntas o secuencia breve de pasos que pueden ser utilizadas en diferentes cursos y para enseñar diferentes contenidos» (Project Zero, s.f., traducción a cargo del autor). Se trata, en definitiva, de protocolos que, al ser de naturaleza pública y repetitiva, contribuyen a hacer el pensamiento visible (Ritchhart, 2002; Perkins, 2003). Algunas de las rutinas más conocidas y empleadas son «Veo-pienso-me pregunto», «Antes pensaba... Ahora pienso...» o «C-S-I» (Color-Símbolo-Imagen). Al ser herramientas de sencilla aplicación, en la mayoría de los casos, no resulta sorprendente que muchos maestros ya las aplicquen en contextos de enseñanza bilingüe. No obstante, al contrario que en el caso de otras estrategias relacionadas con el pensamiento visible -tales como los organizadores gráficos-, su eficacia en contextos AICLE no ha sido aún abordada por la investigación científica.

\section{METODOLOGÍA DE LA INVESTIGACIÓN}

La investigación se desarrolló en dos fases. En la primera, se analizó la idoneidad de las rutinas de pensamiento en el aula bilingüe desde un punto de vista teórico, atendiendo a algunos de los principios fundamentales de la teoría del enfoque AICLE: en concreto, las llamadas «4 $C^{\prime}$ 's» (contenido, comunicación, cognición y cultura) y el principio educativo de andamiaje (scaffolding). En la segunda, se aplicaron sistemáticamente las siete rutinas llamadas fundamentales (core routines) en diferentes asignaturas, todas ellas impartidas en L2 (inglés) en un programa de formación inicial de profesorado. Dicha aplicación se complementó con una serie de intervenciones, más puntuales, en las que pudo explorarse la aplicabilidad de las mismas rutinas a contextos de enseñanza bilingüe en educación primaria, así como el tipo de adaptaciones que es preciso realizar para llevarlas a cabo con éxito.

El estudio tiene un carácter exploratorio, y las conclusiones extraídas de él, lejos de ser generalizables, deben tomarse como punto de partida para estudios cuantitativos y cualitativos que sirvan para articular tanto las fortalezas como las limitaciones de las rutinas de pensamiento en el aula bilingüe.

\section{EXPLORACIÓN TEÓRICA}

\subsection{Cómo visibilizar el pensamiento}

El principal punto de acuerdo entre el marco conceptual del pensamiento visible y el AICLE es que ambos enfoques buscan mejorar la calidad del pensamiento de los alumnos a través del aprendizaje del contenido curricular, así como una mayor visibilidad de estos procesos en el aula. 
Según sostiene Perkins (2003, p.1), el pensamiento «es básicamente invisible». Al contrario que en la práctica deportiva o la danza, por ejemplo, es muy difícil enseñar a pensar proporcionando modelos a imitar por los alumnos. Por eso, resulta imprescindible «visibilizar» los procesos de pensamiento que tienen lugar en el aula. Dicha visibilización puede realizarse de diferentes maneras. Quizá la más importante consiste en documentar el proceso de pensamiento, preguntas, razonamientos, y reflexiones individuales y grupales, lo cual se puede hacer a través de representaciones observables tales como mapas mentales, tablas, listas, diagramas y fichas (Tishman y Palmer, 2005). Podría decirse que dicha representación no es condición suficiente para visibilizar el pensamiento, pero sí necesaria.

La atención al lenguaje que sirve para codificar el pensamiento también es fundamental. Así, se debe promover la utilización explícita en el aula de un léxico del pensamiento (Tishman y Perkins, 1997), con palabras tales como: evidencia, hipótesis, observación, pregunta. De igual forma, es importante priorizar el rol de las preguntas en el aula, especialmente aquellas que fomentan el pensamiento crítico: ¿Qué? ¿Por qué? ¿Y si...? ¿Por qué no...? Todo ello, utilizando el contenido de las asignaturas como objeto de reflexión. Por último, un componente fundamental de la visibilización del pensamiento radica en su carácter social. Las prácticas de pensamiento visible deben dar lugar a momentos de colaboración intelectual en los que, partiendo de las ideas y preguntas de cada individuo, se construya algo más grande, y que debe quedar adecuadamente documentado en las representaciones visuales mencionadas anteriormente (Tishman y Palmer, 2005).

Todas estas recomendaciones sirven para fundamentar el valor de las rutinas de pensamiento, cuya versatilidad y carácter protocolario las hace ideales para tratar contenidos curriculares con un lenguaje de pensamiento y cuestionamiento. Por otro lado, su estructura de fases o etapas, facilita la interacción grupal -pequeños grupos o gran grupo / clase-, así como la representación documental del proceso en organizadores gráficos, listas, plantillas o fichas. Junto con otras prácticas, las rutinas de pensamiento deben contribuir a establecer una «cultura de pensamiento visible» tanto en el aula, como el conjunto del centro educativo (Ritchard, 2002).

\subsection{La «C» de cognición, en AICLE}

Por su parte, el enfoque AICLE también prioriza el pensamiento, tanto en sus principios teóricos como en sus recomendaciones para la práctica docente en el aula. En relación con lo primeros, la cognición es, además del contenido, la comunicación y la cultura, una de las llamadas «4C's» que deben integrar el marco de la programación e impartición de materias bilingües. Los teóricos más influyentes del AICLE son claros al respecto: para que el aprendizaje de contenidos curriculares sea eficaz, el alumno debe involucrarse cognitivamente (cognitively engaged) (2010, p. 29); por tanto, la buena práctica del docente AICLE, debe dirigirse por el desarrollo cognitivo (Mehisto et al., 2008, p. 30).

En su concepción del aprendizaje, el AICLE bebe de fuentes socio-constructivistas. De hecho, Coyle et. al. (2010) acuden al pensamiento de Vygotsky para justificar tanto la necesidad de andamiaje del proceso de aprendizaje, como de la naturaleza esencialmente social del mismo. Como hemos comprobado, ambos postulados son compartidos por el pensamiento visible, lo que sienta las bases teóricas y conceptuales para una compatibilidad entre las actividades recomendadas por el mismo, y el desarrollo de una lección AICLE.

Para concretar la apuesta por la cognición es común, en la programación AICLE, acudir a las taxonomías cognitivas, principalmente la de Bloom (1956) -normalmente en su versión 
revisada por Anderson y Krathwohl (2001)-, pero también a otras, como la de Marzano (2007). Todas estas taxonomías tienen por fin clasificar y ordenar las diferentes destrezas cognitivas. Por ejemplo, en Bloom hallamos, por un lado, las destrezas de orden inferior (LOTS, en inglés): recordar, comprender y aplicar; a su vez, por encima de las anteriores estarían las de orden superior (las HOTS): analizar, evaluar y crear. Pues bien, acudir a una taxonomía cognitiva permite al docente programar objetivos y resultados de aprendizaje en diferentes niveles cognitivos, para no quedarse solo en los de nivel más bajo que es, a menudo y como reportan varios estudios (Hattie, 2012), lo que sucede en muchas aulas. Solo de esta manera puede cumplirse la exigencia teórica del enfoque AICLE de involucrar cognitivamente al discente como parte intrínseca de su aprendizaje de contenidos curriculares.

En definitiva, en este apartado hemos comprobado cómo la calidad cognitiva de las aulas es una preocupación central tanto del pensamiento visible como del AICLE. Además, ambos comparten el postulado socio-constructivista del valor de la comunidad en los procesos de pensamiento, así como la creencia en la necesidad de proveer andamiaje para asegurar el éxito del proceso. Como matices diferenciadores, si bien el pensamiento visible insiste, sobre todo, en sacar a la luz los procesos de reflexión para mejorarlos, en AICLE se atiende más a la clasificación de las destrezas cognitivas para asegurar un aprendizaje de contenidos curriculares cognitivamente rico.

\subsection{El lugar de las rutinas de pensamiento en el aula AICLE}

A continuación, ahondamos en la exploración teórica de la posible compatibilidad entre las rutinas de pensamiento del Proyecto Cero y la enseñanza AICLE, preguntándonos por la conveniencia del uso de las rutinas de pensamiento como recurso de enseñanza-aprendizaje para finalidades concretas. Esta reflexión teórica guiará el diseño de las intervenciones descritas en el apartado tres de este trabajo, por lo que nos centraremos, principalmente, en las siete rutinas llamadas fundamentales.

Seguramente, el uso más común que damos a la mayoría de las rutinas sea la activación cognitiva de los estudiantes al principio de las unidades didácticas, proyectos o, incluso, clases. Como hemos visto, el enfoque AICLE propone una enseñanza de contenido de corte socio-constructivista, en la que el profesor debe conseguir «anclar» los nuevos contenidos que están por enseñar en los esquemas mentales que el alumno ya posee. En palabras de Custodio Espinar: «es fundamental que el docente parta del nivel de desarrollo cognitivo del estudiante, de sus experiencias previas y, en la medida de lo posible, se adapte a su estilo de aprendizaje» (2019, p. 97). Además de las vertientes cognitiva y motivacional, en AICLE la activación también tiene un matiz lingüístico (Mehisto et al., 2008, p. 141): el docente debe también ayudar al estudiante a recordar y reutilizar sus recursos en L2 para acceder a los nuevos contenidos.

Muchas rutinas de pensamiento parecen, a primera vista, ideales para abordar el comienzo de unidades didácticas o proyectos. De las rutinas fundamentales, «Piensaconecta-explora» es, sin duda, la más indicada para esta finalidad. Dicha rutina sirve para «activar conocimientos previos, generar nuevas ideas y conexiones, y despertar la curiosidad para adentrarse en una investigación más profunda» (Fundación Trilema, s.f.). A su vez, las tres preguntas fundamentales de la rutina serían: 1. ¿Qué crees que sabes sobre este tema? 2. ¿Qué ideas o conexiones tienes? 3. ¿Qué cuestiones te invita a explorar? 


\section{Imagen 1. Plantilla para la rutina «Piensa-conecta-explora»}

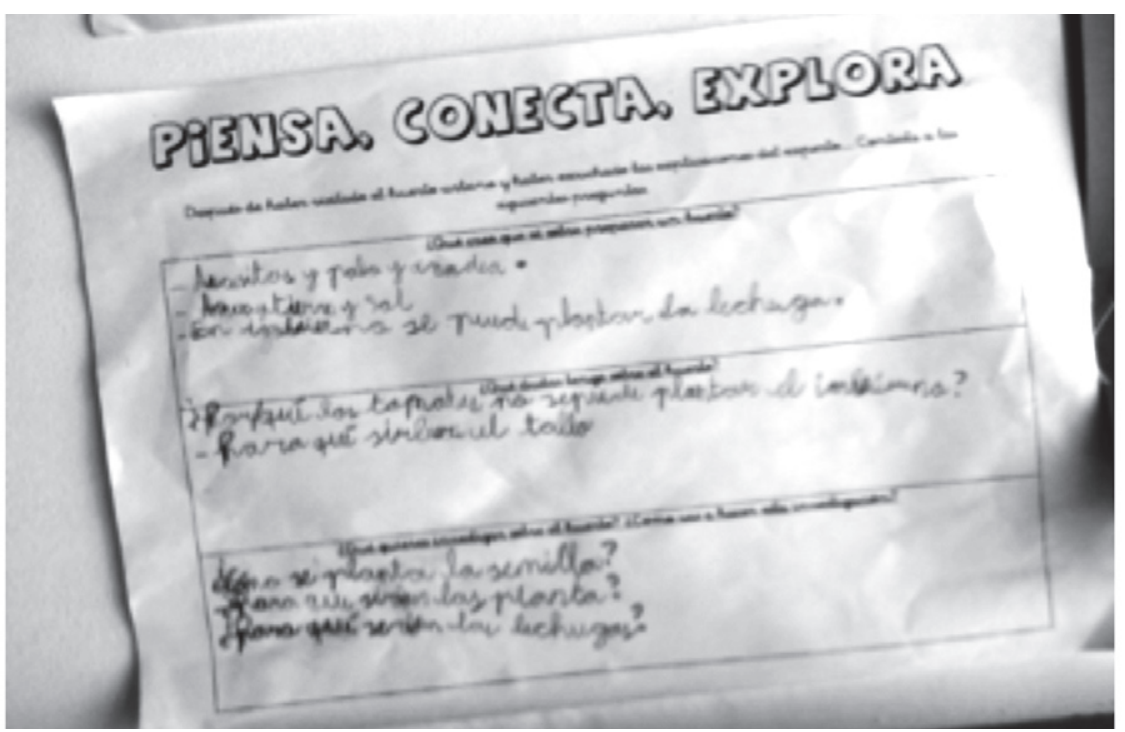

Asimismo, por su apoyo en una imagen, la rutina «Veo-pienso-me pregunto» también es potencialmente excelente para propósitos de activación. Más aún cuando el AICLE recomienda comenzar el aprendizaje con tareas ricas en contexto (context embedded) y no excesivamente demandantes, cognitivamente hablando, para luego ir progresando a actividades más abstractas (Cummins, 2000, p. 79).

Además de la activación previa a la presentación de contenidos, las rutinas pueden servir como estrategias de andamiaje en otros momentos de la clase o de las unidades didácticas. Recordemos que, en AICLE, el concepto de andamiaje adquiere una relevancia especial ya que, además de la comprensión de los contenidos curriculares y el desarrollo de destrezas cognitivas, el docente debe también prever un apoyo a la producción oral y escrita en la lengua extranjera: el llamado andamiaje de transformación, por el que los alumnos procesan el input y lo expresan en un formato diferente, y de producción, por el que crean un producto nuevo a partir de sus conocimientos: una exposición, un póster, un ensayo, etcétera (Dale y Tanner, 2018, p. 36).

Una rutina como Think-Pair-Share, por ejemplo, ayuda a estructurar ejercicios de lectura colaborativa, o actividades de reflexión y comunicación sobre textos, imágenes o experiencias. Igualmente, «Círculo de puntos de vista» puede resultar útil como herramienta de análisis en casos en los que pueden existir una pluralidad de perspectivas sobre un tema o cuestión: debates éticos o políticos, pero también sucesos en obras literarias, que pueden interpretarse de formas diversas por diferentes personajes. Por su parte, «Puntos cardinales» es una rutina más ambiciosa - principalmente, en el tiempo que requiere- y que conecta un análisis de la realidad con la expresión de preocupaciones, ilusiones y necesidades para avanzar. La reservaremos, por tanto, para las fases introductorias de proyectos $\mathrm{u}$ otras estrategias igualmente ambiciosas. 
Por último, y teniendo en cuenta la importancia que otorga el enfoque AICLE a la dimensión metacognitiva del aprendizaje (Berry, 2014), una rutina como «Antes pensaba... Ahora pienso...» parece idónea como instrumento de repaso al final de una clase o unidad. Se trata de una rutina muy sencilla pero rica en metacognición, en la que se incita al alumno a evaluar cómo el aprendizaje ha cambiado su pensamiento sobre un tema concreto.

En definitiva, la naturaleza de muchas de las rutinas de pensamiento las hace aparentemente compatibles con las estrategias y los principios metodológicos recomendados en AICLE. Sin embargo, para confirmar esta compatibilidad, es necesario probarlas sobre el terreno. No solo es importante evaluar si este recurso mejora otros de práctica habitual en AICLE; también lo es analizar hasta qué punto los límites impuestos por una menor competencia lingüística, en L2 que en L1, puede comprometer su eficacia.

\section{LECCIONES DE LA PRÁCTICA: APLICACIÓ DE LAS RUTINAS DE PENSAMIENTO FUNDAMENTALES EN CONTEXTOS BILINGÜES}

A continuación, se presentan y analizan los resultados la aplicación sistemática de siete rutinas de pensamiento con estudiantes de los grados de Maestro de Educación Infantil y Maestro de Educación Primaria, en el Centro de Estudios Superiores «Don Bosco» (adscrito a la Universidad Complutense de Madrid). Se complementan con varias aplicaciones de algunas de las mismas rutinas en tres colegios de la Comunidad de Madrid (España), dos de ellos adscritos a su programa bilingüe. Las rutinas de pensamiento elegidas fueron las llamadas rutinas fundamentales (core routines). Son rutinas fáciles de aplicar e implementar, pero ofrecen la suficiente variedad en el tipo de pensamiento que facilita.

\section{1. Ámbito y método de aplicación}

La tabla 1 recoge el objetivo y las condiciones de aplicación de cada rutina con estudiantes universitarios de los grados de Maestro. Estas intervenciones se llevaron a cabo a lo largo de tres cursos académicos -entre 2014 y 2017-, interactuando siempre en lengua inglesa. En este sentido, aunque el nivel de inglés de los grupos era heterogéneo, podemos situarlo, con carácter orientativo, en B1-B2 para los cursos, $1^{\circ}$ y $2^{\circ}$ y B2-C1 para los de cuarto, utilizando la escala del Marco Común Europeo de Referencia para las Lenguas (MCERL). En cuanto al número de participantes por rutinas, estarían entre 15 y 35. Todas fueron realizadas con más de un grupo y, salvo las más ambiciosas (rutinas 4 y 7), todas se llevaron a cabo al menos dos veces con cada grupo, para que los estudiantes pudieran así experimentar su aspecto de «rutina». 
Tabla 1. Rutinas de pensamiento aplicadas en clases de la modalidad bilingüe, de los grados de Maestro. CES Don Bosco, 2014-17

\begin{tabular}{|c|c|c|}
\hline Nombre de la rutina & Curso / asignatura & Uso \\
\hline $\begin{array}{l}\text { 1. ¿Qué te hace decir } \\
\text { eso? }\end{array}$ & Todos. & $\begin{array}{l}\text { Rutina utilizada como pregunta frecuente en el } \\
\text { aula, para animar a los alumnos a justificar sus } \\
\text { puntos de vista. }\end{array}$ \\
\hline \multirow[t]{2}{*}{$\begin{array}{l}\text { 2. Piensa, conecta, } \\
\text { explora. }\end{array}$} & \multirow[t]{2}{*}{$\begin{array}{l}4^{\circ} \text { Formación para } \\
\text { el bilingüismo. }\end{array}$} & $\begin{array}{l}\text { Reflexión inicial sobre el concepto de } \\
\text { scaffolding (andamiaje) en AICLE. }\end{array}$ \\
\hline & & $\begin{array}{l}\text { Reflexión inicial sobre la enseñanza bilingüe (en } \\
\text { inglés) en educación primaria en la Comunidad } \\
\text { de Madrid. }\end{array}$ \\
\hline \multirow[t]{2}{*}{ 3. Think Pair Share. } & \multirow[t]{2}{*}{$\begin{array}{l}2^{\circ} \text { Fundamentos } \\
\text { de las Ciencias } \\
\text { Sociales. }\end{array}$} & $\begin{array}{l}\text { Reflexión sobre las experiencias de los } \\
\text { estudiantes, durante su período de prácticas, en } \\
\text { relación con los contenidos de la asignatura. }\end{array}$ \\
\hline & & $\begin{array}{l}\text { Lluvia de ideas colaborativa sobre la pregunta: } \\
\text { ¿En qué consiste la investigación histórica? }\end{array}$ \\
\hline $\begin{array}{l}\text { 4. Círculo de puntos } \\
\text { de vista. }\end{array}$ & $\begin{array}{l}2^{\circ} \text { Lectura y } \\
\text { escritura creativa. }\end{array}$ & $\begin{array}{l}\text { Reflexionar sobre el personaje de un } \\
\text { relato o poema, poniéndose en el lugar de } \\
\text { otros personajes del mismo (desarrollo del } \\
\text { pensamiento crítico). }\end{array}$ \\
\hline \multirow[t]{2}{*}{$\begin{array}{l}\text { 5. Antes pensaba... } \\
\text { Ahora pienso... }\end{array}$} & \multirow[t]{2}{*}{$\begin{array}{l}4^{\circ} \text { Formación para } \\
\text { el bilingüismo. }\end{array}$} & $\begin{array}{l}\text { Repaso de lo aprendido sobre los diferentes } \\
\text { programas de enseñanza bilingües. }\end{array}$ \\
\hline & & $\begin{array}{l}\text { Repaso de lo aprendido sobre los organizadores } \\
\text { gráficos en contextos de enseñanza bilingüe. }\end{array}$ \\
\hline \multirow[t]{2}{*}{$\begin{array}{l}\text { 6. Veo, pienso, me } \\
\text { pregunto. }\end{array}$} & \multirow[t]{2}{*}{$\begin{array}{l}1^{0} \text { Sociología de la } \\
\text { Educación. }\end{array}$} & $\begin{array}{l}\text { Análisis de una imagen en la que se ven } \\
\text { maestras, padres y niños en un aula de } \\
\text { educación infantil. }\end{array}$ \\
\hline & & $\begin{array}{l}\text { Reflexión sobre los conceptos de cultura, } \\
\text { estatus social y símbolos a partir de una imagen } \\
\text { de unas personas en un parque de la ciudad. }\end{array}$ \\
\hline \multirow[t]{2}{*}{ 7. Puntos cardinales. } & $\begin{array}{l}2^{\circ} \text { Lectura y } \\
\text { escritura creativa. }\end{array}$ & $\begin{array}{l}\text { Reflexión inicial sobre un proyecto de creación } \\
\text { y edición de una revista literaria en línea. }\end{array}$ \\
\hline & $\begin{array}{l}2^{\circ} \text { Fundamentos } \\
\text { de las Ciencias } \\
\text { Sociales. }\end{array}$ & $\begin{array}{l}\text { Reflexión sobre la posibilidad de llevar a cabo } \\
\text { un aprendizaje basado en proyectos (ABP), en } \\
\text { la práctica docente futura. }\end{array}$ \\
\hline
\end{tabular}


En relación con la forma de dirigir las rutinas - un aspecto clave para su eficacia- se siguieron las recomendaciones de los formadores de la Fundación Trilema (España), liderada por la filósofa y pedagoga Carmen Pellicer, que ofrece numerosos cursos y acciones formativas sobre esta y otras herramientas didácticas. En concreto:

- Se procuró que todos los estudiantes participaran en cada rutina, aunque dejando siempre la opción de renunciar al turno de palabra.

- En la mayoría de los casos, la rutina se realizó con apoyo de un soporte escrito: anotaciones del estudiante en su cuaderno, apuntes en la pizarra, o fichas y plantillas diseñadas para reflejar los pasos de la rutina en cuestión.

- El profesor repetía los comentarios de los estudiantes en voz alta, asegurándose de que las ideas llegaran al conjunto del grupo, que a veces podía ser muy numeroso (35 estudiantes).

Como complemento del análisis, se realizaron una serie de observaciones e intervenciones en clases de educación primaria de tres colegios de la Comunidad de Madrid: El Colegio Mater Immaculata (Madrid), el Colegio Virgen de Mirasierra (Madrid) y el CEIP Aldebarán (Tres Cantos). El objetivo de estas intervenciones fue el evaluar el valor motivacional de las rutinas con estudiantes de diferentes grupos de primaria, así como tener una idea más ajustada de cómo adaptarlas a la competencia lingüística de los niños en estas edades. Las intervenciones se llevaron a cabo bien por el autor de este artículo, por un profesor colaborador, o por una estudiante de $4^{\circ}$ curso del grado de Maestro de Educación Primaria, que aplicaba las rutinas como parte de su Trabajo de Fin de Grado. En todos los casos, las rutinas se realizaron según las directrices señaladas con anterioridad. La tabla 2 resume las rutinas de pensamiento, aplicadas por el equipo investigador en estos tres centros.

Tabla 2. Rutinas de pensamiento aplicadas en centros de educación primaria

\begin{tabular}{|l|l|l|}
\hline \multicolumn{1}{|c|}{ Nombre de la rutina } & \multicolumn{1}{|c|}{ Curso / asignatura } & \multicolumn{1}{c|}{ Uso } \\
\hline Piensa, conecta, explora. & $\begin{array}{l}2^{0} \text { Educación primaria, } \\
\text { Natural Science. }\end{array}$ & $\begin{array}{l}\text { Se utiliza al principio de una unidad } \\
\text { didáctica sobre el sistema solar, como rutina } \\
\text { de activación, para repasar conocimientos } \\
\text { previos y hacerse preguntas que guíen el } \\
\text { futuro aprendizaje. Soporte documental: } \\
\text { post-its pegados en un mural colaborativo. }\end{array}$ \\
\hline Think Pair Share. & $\begin{array}{l}4^{0} \text { Educación Primaria, } \\
\text { Social Science. }\end{array}$ & $\begin{array}{l}\text { Se utiliza la rutina como actividad de } \\
\text { post-lectura basada en un texto sobre la } \\
\text { extinción de los grandes mamíferos del } \\
\text { Paleolítico. La rutina promueve la escucha } \\
\text { activa y facilita la conversación en lengua } \\
\text { inglesa. Soporte documental: post-its. }\end{array}$ \\
\hline $\begin{array}{l}\text { Círculo de puntos de } \\
\text { vista. }\end{array}$ & $\begin{array}{l}4^{0} \text { Educación primaria, } \\
\text { Arts and Crafts. }\end{array}$ & $\begin{array}{l}\text { Se utiliza la rutina para reflexionar sobre un } \\
\text { cuadro desde la perspectiva de diferentes } \\
\text { personas que aparecen en él. Soporte } \\
\text { documental: escritura individual de los } \\
\text { alumnos en sus cuadernos. }\end{array}$ \\
\hline
\end{tabular}




\begin{tabular}{|l|l|l|}
\hline Veo, pienso, me pregunto. & $\begin{array}{l}2^{0} \text { Educación primaria. } \\
\text { English. }\end{array}$ & $\begin{array}{l}\text { Se utiliza la imagen de un cuento para } \\
\text { generar hipótesis sobre el contenido del } \\
\text { cuento, previamente a su lectura. Soporte } \\
\text { documental: ninguno; actividad realizada de } \\
\text { forma oral, en asamblea. }\end{array}$ \\
\cline { 2 - 3 } & $\begin{array}{l}1^{\circ} \text { Educación primaria, } \\
\text { Natural Science }\end{array}$ & $\begin{array}{l}\text { Los estudiantes aplican los pasos de la } \\
\text { rutina a la imagen de su animal favorito. La } \\
\text { rutina sirve para repasar los contenidos de } \\
\text { la unidad, así como preparar una exposición } \\
\text { oral. Soporte documental: organizador } \\
\text { gráfico con los pasos de la rutina. }\end{array}$ \\
\hline
\end{tabular}

\subsection{Análisis de la eficacia de las rutinas fundamentales, en AICLE}

La aplicación sistemática de las siete rutinas fundamentales en los ámbitos detallados anteriormente nos permitió obtener una serie de conclusiones preliminares que, teniendo en cuenta el carácter exploratorio de este estudio, deben contribuir a formular hipótesis para estudios más exhaustivos.

\subsubsection{Fortalezas}

Con carácter general, las rutinas de pensamiento demuestran una serie de fortalezas especialmente relevantes en contextos AICLE. La primera, como cabría suponer, es su simplicidad y carácter protocolario que facilita que los alumnos comiencen la rutina sin necesitar apenas de explicación previa. Algo que resulta especialmente importante en enseñanza en L2, ya que la repetición de una actividad -el que se convierta en rutina- evita el no siempre sencillo proceso de proporcionar instrucciones claras en la lengua extranjera. Como apunta Salmon (2010, p. 137), el carácter predecible de las rutinas supone un andamiaje importante en situaciones en donde la adquisición de la lengua está en juego, tales como en Educación Infantil o, en el caso que nos ocupa, de enseñanza bilingüe.

Por otro lado, el lenguaje formulaico y repetitivo de las mismas actúa como otro importante apoyo para las intervenciones orales de los alumnos; sobre todo, de aquellos que tienen menor competencia lingüística en L2. Dicho lenguaje, sumado a la práctica habitual de que los alumnos escriban sus respuestas en post-its, cuaderno u organizadores gráficos, facilita un entorno dialógico mucho más inclusivo. Por ejemplo, la realización de una rutina «Veo-pienso-me pregunto» a partir de una imagen, tenderá a ser más eficaz para más estudiantes de la clase, que una pregunta abierta sobre la misma imagen, que suscitará respuestas rápidas solo de unos pocos. 


\section{Imagen 2. Ficha sobre la rutina «Veo-pienso-me pregunto», anotada por un estudiante de $1^{\circ}$ de educación primaria}

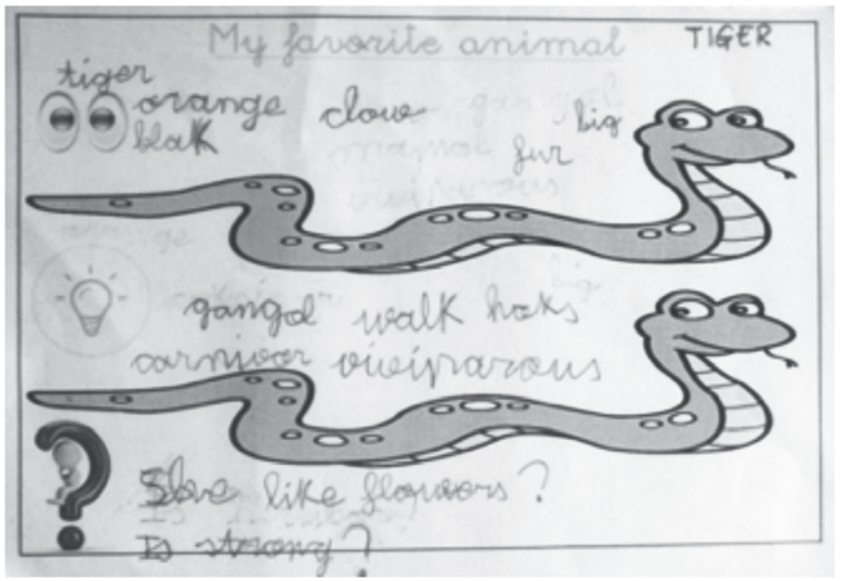

Fuente: Colegio Virgen de Mirasierra, Madrid.

Desde luego, en un sentido más amplio, el empleo de rutinas de pensamiento en el aula de AICLE puede contribuir a evitar que la competencia lingüística penalice a algunos estudiantes y se convierta, de forma inadvertida, en un componente «invisible» de la evaluación. Como señala Morton (2019, p.13), el docente AICLE puede estar tentado a otorgar mejores notas a alumnos que escriben y hablan con más fluidez, o con menores errores gramaticales o de ortografía. Aparte de un diseño más eficiente de técnicas de evaluación formativa y sumativa, las rutinas de pensamiento permiten escuchar mejor las ideas y reflexiones de todos los estudiantes, independientemente de su nivel de competencia lingüística.

Una segunda fortaleza de muchas de las rutinas, es que exigen a los estudiantes que formulen preguntas. Aparte de contribuir a visibilizar el pensamiento (Tishman y Palmer, 2005) y fomentar que el alumno lidere su proceso de aprendizaje (Walsh y Sattes, 2017), las rutinas permiten a los estudiantes practicar unas estructuras gramaticales de gran dificultad lingüística en L2: preguntas con pronombres interrogativos (las llamadas Wh-questions), preguntas de sí/no, preguntas indirectas. Son preguntas de una gramática compleja -más aún para un estudiante de educación primaria- y rutinas tales como «Veo-pienso-me pregunto» $\mathrm{o}$ «Piensa-conecta-explora» pueden contribuir eficazmente a su afianzamiento.

Por último, nuestras intervenciones parecen confirmar la hipótesis de que las rutinas de pensamiento promueven una reflexión más personalizada. Un buen ejemplo de ello es la rutina «Antes pensaba... Ahora pienso...», de gran valor metacognitivo, que pide al estudiante que explicite cómo el aprendizaje de una clase, unidad o un curso entero ha afectado su pensamiento sobre un tema determinado.

\subsubsection{Limitaciones}

El valor de muchas de las rutinas de pensamiento, para fomentar a reflexión y la comunicación en el aula de AICLE, no debe oscurecer algunas de sus limitaciones más importantes, mismas que quedaron patentes en nuestro proceso de aplicación sistemática. 
En relación con la calidad cognitiva del aprendizaje, las rutinas pueden complementar - pero nunca sustituir- otras actividades más ambiciosas, tales como proyectos, experimentos o, incluso, el procesamiento de textos u otros inputs a través de la utilización de organizadores gráficos. Lo apuntábamos antes: muchas de las rutinas hallan su entorno natural en momentos de activación del aprendizaje en los que, según la matriz de Cummins (2000), se recomienda que las actividades sean por un lado ricas en contexto y, por otro, de baja exigencia cognitiva.

En nuestra experiencia, las rutinas pueden ser excelentes para clarificar o visibilizar procesos de reflexión: por ejemplo, hacer que los alumnos sean conscientes de la diferencia entre un juicio sobre algo y las evidencias en las que dicho juicio se funda. Sin embargo, estos sencillos protocolos, de por sí, no aseguran el desarrollo de destrezas cognitivas de orden superior. Incluso una rutina de gran valor para el desarrollo del pensamiento crítico como "Círculo de puntos de vista», solo tiene valor en tanto que facilitadora de la apertura mental ante una pluralidad de perspectivas, necesitando de reflexiones y actividades posteriores en las que el alumno compare, analice, discrimine, evalúe, etcétera.

Algo similar sucede en el ámbito lingüístico. La repetición de rutinas puede dar la impresión al docente de que los estudiantes están trabajando de forma incesante la competencia oral en la L2, satisfaciendo así la «C» de comunicación del enfoque AICLE. Pero esa impresión solo es cierta en parte. Aparte de la rutina Think-Pair-Share, más propicia a una verdadera comunicación entre varios alumnos, la mayoría de las rutinas carece de los componentes fundamentales de una actividad comunicativa, a saber: capacidad de elección en cuanto al contenido y el lenguaje (choice), respuesta del interlocutor (feedback) y, sobre todo, la necesidad de escuchar para obtener información u opinión del interlocutor (information/opinion gap) (Larsen-Freeman, 2000). Al igual que sucede con la cognición, para cumplir con las expectativas del enfoque AICLE, en relación con la comunicación, no bastará con las rutinas, sino que habrá que proponer a los alumnos actividades de mucha mayor riqueza lingüística y, lo que es fundamental en AICLE, que atiendan a funciones, géneros y productos de comunicación académica en general, así como específicos del área curricular que se está trabajando en L2 (ver numerosos ejemplos de ello en [López Hernández, 2015]).

Por último, la aplicación habitual de rutinas de pensamiento en entornos de aprendizaje cooperativo nos ofrece dudas. Como hemos comprobado, salvo en el caso de ThinkPair-Share, la principal dinámica de interacción en las rutinas es la del individuo con el conjunto del grupo-case. En aprendizaje cooperativo, sin embargo, los estudiantes trabajan en grupos más o menos estables -cambiando cada mes, por ejemplo- y con roles concretos asignados. De hecho, el aprendizaje cooperativo tiene ya una serie de estrategias que fomentan el procesamiento de la información: lápices al centro, folio giratorio, 1-2-4, grupos de expertos, etcétera (Cooperativa, s.f.). Estas y otras estrategias de trabajo comunes en entornos de trabajo cooperativo tienen, en cierta forma, el carácter de rutina, por lo que, en caso de querer aplicar las rutinas del Proyecto Cero, el docente y su equipo deberán ser especialmente cuidadosos en el análisis del valor añadido que aporten a su dinámica de trabajo.

Como complemento a este análisis general, se incluye a continuación una tabla que resume, de forma esquemática, nuestra valoración de la idoneidad de cada rutina fundamental en entornos AICLE. 
Tabla 3. Resumen de la conveniencia del uso de cada rutina "Fundamenta», en un entorno AICLE

\begin{tabular}{|c|c|c|}
\hline Nombre de la rutina & Fortalezas (F) / Limitaciones (L) & $\begin{array}{l}\text { Recomendaciones para su uso } \\
\text { en AICLE }\end{array}$ \\
\hline ¿Qué te hace decir eso? & $\begin{array}{l}\text { F: Pregunta ágil, más natural en inglés } \\
\text { que en español. }\end{array}$ & $\begin{array}{l}\text { Usar como pregunta habitual } \\
\text { de clase, para fomentar un } \\
\text { pensamiento basado en } \\
\text { evidencias. }\end{array}$ \\
\hline $\begin{array}{l}\text { Piensa, conecta, } \\
\text { explora. }\end{array}$ & $\begin{array}{l}\text { F: La segunda fase (puzzle) sirve para } \\
\text { que los alumnos formulen preguntas } \\
\text { en inglés. L: La tercera fase se parece } \\
\text { demasiado a la segunda, y en muchos } \\
\text { casos, resulta redundante. }\end{array}$ & $\begin{array}{l}\text { Excelente rutina de activación } \\
\text { al principio de una unidad } \\
\text { didáctica. Muy parecida a la } \\
\text { estrategia K-W-L, de uso más } \\
\text { habitual en AICLE. }\end{array}$ \\
\hline Think Pair Share. & $\begin{array}{l}\text { F: Fomenta la comunicación auténtica } \\
\text { (con information gap). Muy versátil, ya } \\
\text { que se puede utilizar para reflexionar } \\
\text { sobre una imagen, lectura, tema, } \\
\text { opinión... }\end{array}$ & $\begin{array}{l}\text { Mejor omitir en entornos de } \\
\text { trabajo cooperativo, que ya } \\
\text { ofrece la estrategia } 1-2-4 \text {, muy } \\
\text { similar. }\end{array}$ \\
\hline $\begin{array}{l}\text { Círculo de puntos de } \\
\text { vista. }\end{array}$ & $\begin{array}{l}\text { F: Fomenta el pensamiento crítico. L: } \\
\text { Requiere mucho tiempo; no es fácil } \\
\text { encontrar textos u obras de arte que } \\
\text { contengan tantas perspectivas. }\end{array}$ & $\begin{array}{l}\text { Se puede trabajar en parejas, } \\
\text { en caso de que el grupo } \\
\text { sea numeroso o el texto sea } \\
\text { complejo. }\end{array}$ \\
\hline $\begin{array}{l}\text { Antes pensaba... Ahora } \\
\text { pienso... }\end{array}$ & $\begin{array}{l}\text { F: Personaliza el aprendizaje. Recicla } \\
\text { la estructura used to, que no es } \\
\text { de fácil aplicación. L: No todas las } \\
\text { temáticas se prestan a ello. }\end{array}$ & $\begin{array}{l}\text { Difícil de realizar en los cursos } \\
\text { más bajos de educación } \\
\text { primaria, ya que el alumno no } \\
\text { siempre recuerda cuál era su } \\
\text { punto de partida. }\end{array}$ \\
\hline $\begin{array}{l}\text { Veo, pienso, me } \\
\text { pregunto. }\end{array}$ & $\begin{array}{l}\text { F: Muy versátil; aporta andamiaje a la } \\
\text { producción oral en L2. Excelente para } \\
\text { desarrollar fluidez en la formulación } \\
\text { de preguntas en L2. }\end{array}$ & $\begin{array}{l}\text { Se puede usar como rutina de } \\
\text { activación, pero también como } \\
\text { repaso. }\end{array}$ \\
\hline Puntos cardinales. & $\begin{array}{l}\text { F: Incluye el aspecto emocional } \\
\text { además del cognitivo. L: Es difícil } \\
\text { realizarla bien con estudiantes de } \\
\text { un nivel bajo de inglés (B1 MCER o } \\
\text { inferior). }\end{array}$ & $\begin{array}{l}\text { Ideal para reflexionar sobre un } \\
\text { proyecto o tarea que vaya a } \\
\text { alargarse en el tiempo. Por su } \\
\text { relativa complejidad, conviene } \\
\text { que los alumnos ya la hayan } \\
\text { realizado en L1. }\end{array}$ \\
\hline
\end{tabular}

\subsection{Recomendaciones para la adaptación de las RRPP a contextos AICLE}

Las numerosas intervenciones realizadas nos permiten ofrecer una serie de recomendaciones prácticas sobre cómo adaptar las rutinas de pensamiento en un entorno AICLE, en donde la participación en las rutinas podría penalizarse por una deficiente competencia en lengua inglesa. 
- Dejar suficiente tiempo de preparación de las respuestas. Con alumnos que poseen cierta fluidez en la escritura -segundo o tercero de primaria en adelante-, las fichas, plantillas y los organizadores gráficos son de gran ayuda, puesto que facilitan que todos intervengan, sin sentirse cohibidos en caso de tener menos recursos lingüísticos en L2.

- Maximizar la exposición del alumno a la palabra escrita,como recordatorio del léxico empleado, pero también para fijar la correcta ortografía de los términos. Se trata de un ejercicio de andamiaje lingüístico muy beneficioso, teniendo en cuenta la dificultad de la ortografía en lengua inglesa.

- No forzar a ningún alumno a hablar en caso de que no tenga nada que decir, o su idea o comentario ya haya sido expresado por algún otro compañero.

- Animar a los alumnos a utilizar las frases asociadas a cada etapa de la rutina, por ejemplo: I think that...; My question is... Esto es incluso más importante en L2 que en L1, debido a los menores recursos lingüísticos de los que dispone el estudiante.

- Al escuchar la intervención de los alumnos, es buena idea repetir sus palabras, reformulándolas en caso de que contengan algún error léxico o gramatical. Así la idea llega mejor a toda la clase, que a la vez recibe un modelo lingüístico correcto.

- En general, es mejor hacer menos rutinas diferentes, pero con más repeticiones, que más rutinas con menos repeticiones. El proceso de entender y aplicar una rutina requiere un esfuerzo que se multiplica al trabajar en L2. En este sentido, una estrategia muy exitosa es coordinar la aplicación de rutinas específicas por clase o curso entre varios profesores, para asegurar la interiorización y mecanización de las mismas por parte de los alumnos.

\section{CONCLUSIONES}

A través de este estudio teórico y aplicado, ha quedado demostrado que existe una gran compatibilidad entre los principios del pensamiento visible y los del AICLE. En concreto, las rutinas de pensamiento del Proyecto Cero de Harvard - $u$ otras similarespueden contribuir de forma eficaz al desarrollo de la cognición y la comunicación en entornos AICLE. Como hemos visto, las rutinas de pensamiento fomentan una mayor inclusión de los alumnos en las tareas de reflexión grupal. Bien aplicadas, minimizan el efecto penalizador de las limitaciones en la competencia lingüística en L2. Además, demuestran ser instrumentos de interacción especialmente ágiles, ya que su carácter repetitivo minimiza el tiempo dedicado a dar instrucciones sobre su realización. A nivel lingüístico, muchas de las rutinas permiten a los estudiantes desarrollar una gran fluidez a la hora de hacer preguntas, lo cual no es sencillo en lengua inglesa.

Sin embargo, el uso de las rutinas de pensamiento - al menos, las fundamentales- no asegura una calidad suficiente de la clase ni a nivel cognitivo ni lingüístico. Para ello, se deberá proponer a los estudiantes, la realización de actividades y proyectos más ambiciosos y exigentes que les lleven, por un lado, a desarrollar destrezas cognitivas de orden superior y, por otro, a trabajar con éxito funciones, géneros y productos, orales y escritos. 
A partir de estas conclusiones preliminares sobre la idoneidad de las rutinas de pensamiento en entornos AICLE, se recomienda que futuras investigaciones amplíen el alcance a todas las rutinas de pensamiento, no solo las fundamentales. Asimismo, sería interesante responder preguntas de investigación tales como:

- ¿Cuáles son las rutinas de pensamiento más empleadas por los docentes AICLE? ¿Qué razones impulsan a los docentes a utilizarlas, en general? ¿Para qué fines concretos se emplea cada una?

- ¿En qué asignaturas AICLE se usan rutinas con mayor asiduidad?

- ¿Cómo se han formado, en rutinas de pensamiento, los profesores que las emplean?

- ¿Promueven las rutinas de pensamiento, también en otras actividades, una participación más activa de los estudiantes más introvertidos o con menor nivel de inglés?

- ¿Cómo pueden adaptarse eficazmente las rutinas de pensamiento a contextos de trabajo cooperativo con grupos estables?

\section{REFERENCIAS}

Anderson, L. W. \& Krathwohl, D. R. (Eds.) (2001). A taxonomy for learning, teaching, and assessing: A revision of Bloom's taxonomy of educational objectives. Boston, MA: Allyn \& Bacon.

Berry, M. (2014) Metacognition in CLIL. CLIL Magazine, Spring 2014. Recuperado de https: / / clilmedia.com/wp-content/uploads/2015/05/CLIL-Magazine-Spring-2014.pdf

Coyle, D., Hood, P. \& Marsh, D. (2010). Content and Language Integrated Learning. Cambridge, United Kingdom: Cambridge University Press.

Cooperativa de Enseñanza José Ramón Otero (s.f.). Aprendizaje cooperativo. Propuesta para la implantación de una estructura de cooperación en el aula. Recuperado de http: / / labmadrid.com/aprendizaje-cooperativo/

Cummins, J. (2000). Language, Power and Pedagogy: Bilingual Children in the Crossfire. Blue Ridge Summit, PA Multilingual Matters

Custodio Espinar, M. (2019). Los principios metodológicos AICLE (aprendizaje integrado de contenidos y lengua). Madrid: Fundación Universitaria Española.

Dale, L., Tanner, R. \& Thornbury, S. (2018). CLIL activities: A resource for subject and language teachers. Cambridge: Cambridge University Press.

Fundación Trilema (s.f.). Rutinas de pensamiento. Repaso a las rutinas fundamentales. Recuperado de https: / / es.slideshare.net/ FundacionTrilema/rutinas-de-pensamiento-rutinas-fundamentales 
Graddol, D. (2006). English next (vol. 62). Londres: British Council.

Hattie, J. (2012). Visible Learning for teachers. Maximizing impact on learning. Abingdon and New York: Routledge.

Larsen-Freeman, D. (2000). Techniques and principles of language teaching (2nd ed.) Oxford, UK: Oxford University Press.

López Hernández, A. (Coord.). (2015). Propuestas de scaffolding en enseñanza universitaria bilingüe. Educación y futuro, 32, 117-165. Recuperado de https:/ / cesdonbosco. com/documentos/revistaeyf/EYF_32.pdf

Marzano, R.J. \& Kendall, J.S. (2007). The new taxonomy of educational objectives. Thousand Oaks, CA: Corwin Press.

McDougald, Jermaine. (2015). Teachers' attitudes, perceptions and experiences in CLIL: A look at content and language. Colombian Applied Linguistics Journal, 17, 25-41. https: / / doi.org/10.14483/udistrital.jour.calj.2015.1.a02

Mehisto, P., Marsh, D. \& Frigols, M. J. (2008). Uncovering CLIL: Content and language integrated learning in bilingual and multilingual education. Oxford: Macmillan Education.

Morton, T. (2019). La evaluación en AICLE: dificultades y oportunidades. Padres y Maestros/Journal of Parents and Teachers, 378, 11-18. https: / / doi.org/10.14422 / pym.i378. y2019.002

Perkins, D.N. (2003). Making Thinking Visible. Project Zero. Traducción española disponible en: http: / / conexiones.dgire.unam.mx/wp-content/uploads/2017/11/perkins_david_como_hacer_visible_el_pensamiento.pdf

Project Zero at the Harvard Graduate School of Education (s.f.). Visible Thinking. Recuperado de https:/ / pz.harvard.edu/ projects / visible-thinking

Ritchhart, R. (2002). Intellectual character: What it is, why it matters, and how to get it. San Francisco: Jossey-Bass.

Salmon, A.K. (2010). Engaging Young Children in Thinking Routines. Childhood Education, 86(3), 132-137. https:/ / doi.org/10.1080/00094056.2010.10523133

Tishman, S. \& Palmer, P. (2005) Visible Thinking. Leadership Compass 2(4)

Tishman, S. \& Perkins, D. (1997). The language of thinking. Phi Delta Kappan, 78(5), 368.

Walsh, J. A. \& Sattes, B. D. (2017). Quality questioning: Research-based practice to engage every learner. Thousand Oaks, California: Corwin. 